\title{
ASYMPTOTIC BEHAVIOUR OF IDEALS RELATIVE TO INJECTIVE MODULES OVER COMMUTATIVE NOETHERIAN RINGS
}

\author{
by H. ANSARI TOROGHY and R. Y. SHARP
}

(Received 11th July 1989)

\begin{abstract}
Let $E$ be an injective module over the commutative Noetherian ring $A$, and let $a$ be an ideal of $A$. The $A$-module $\left(0: E^{\mathfrak{a})}\right.$ has a secondary representation, and the finite set $A_{t t}(0: E)$ of its attached prime ideals can be formed. One of the main results of this note is that the sequence of sets $\left(A_{t t}\left(0:_{E} a^{n}\right)\right)_{n \in N}$ is ultimately constant. This result is analogous to a theorem of $M$. Brodmann that, if $M$ is a finitely generated $A$-module, then the sequence of sets $\left(\operatorname{Ass}_{A}\left(M / a^{n} M\right)\right)_{n \in N}$ is ultimately constant.
\end{abstract}

1980 Mathematics subject classification (1985 Revision): 13C11, 13E05

\section{Introduction}

It is a well-known result of $\mathrm{M}$. Brodmann [1] that, if $M$ is a finitely generated module over the commutative Noetherian ring $A$ (with identity) and $a$ is an ideal of $A$, then the sequence of sets $\left(\operatorname{Ass}_{A}\left(M / \mathfrak{a}^{n} M\right)\right)_{n \in N}$ is ultimately constant, that is $\operatorname{Ass}_{A}\left(M / \mathfrak{a}^{n} M\right)$ is, for all sufficiently large $n$, independent of $n$. (We use $\mathbb{N}$ (respectively $\mathbb{N}_{0}$ ) to denote the set of positive (respectively non-negative) integers.) It is a very easy consequence of this result of Brodmann that the same conclusion holds of we relax the hypotheses and assume only that $A$ is a commutative ring (with identity) and $M$ is a Noetherian $A$-module.

In [8], the present second author established a dual result for an Artinian module $N$ over a commutative ring $R$ (with identity). This dual result was phrased in terms of the notions of secondary representations and attached prime ideals of Artinian $R$-modules (see $[3,2$ or 6]), and showed that, if $I$ is an ideal of $R$, then the sequence of sets $\left(\operatorname{Att}_{R}\left(0:_{N} I^{n}\right)\right)_{n \in N}$ is ultimately constant.

Every Artinian $R$-module possesses a secondary representation. (It is convenient to take the view that the zero $R$-module is the sum of the empty family of its secondary submodules.) Now it was shown in [7] that the class of all $R$-modules which possess secondary representations can be more extensive than the class of all Artinian $R$ modules: indeed, when we take the commutative Noetherian ring $A$ for $R$, every injective $A$-module possesses a secondary representation [7, Theorem 2.3]. We shall see below that the arguments of that paper can easily be modified to show that, when $A$ is Noetherian, $E$ is an injective $A$-module and $\mathfrak{a}$ is an ideal of $A$, the submodule $\left(0:_{E} \mathfrak{a}\right)$ of $E$ has a secondary representation, and so we can form the finite set $\operatorname{Att}_{A}(0: E)$ of its attached prime ideals. One of the main results of this note is that, in these 
circumstances, the sequence of sets $\left(\operatorname{Att}_{A}\left(0:_{E} \mathfrak{a}^{n}\right)\right)_{n \in N}$ is ultimately constant; in addition, we shall obtain other results which are reminiscent of facts concerning the asymptotic behaviour of an ideal $I$ of $R$ relative to an Artinian module $N$ over $R$.

Throughout the remainder of this paper, $A$ will denote a (non-trivial) commutative Noetherian ring with identity; the symbol $R$ will denote a commutative ring (with identity) which is not necessarily Noetherian. We shall use the notation and terminology of [7] and [8] concerning secondary representations and attached prime ideals. We shall also use the notation $\operatorname{Occ}(E)$ of $[7$, Section 2] in connection with an injective $A$ module $E$ : this is explained as follows. By well-known work of Matlis and Gabriel, there is a family $\left(\mathfrak{p}_{\alpha}\right)_{\alpha \in \Lambda}$ of prime ideals of $A$ for which $E \cong \bigoplus_{\alpha \in \Lambda} E\left(A / \mathfrak{p}_{\alpha}\right)$ (we use $E(L)$ to denote the injective envelope of an $A$-module $L$ ), and the set $\left\{\mathfrak{p}_{\alpha}: \alpha \in \Lambda\right\}$ is uniquely determined by $E$ : we denote it by $\operatorname{Occ}(E)$.

\section{Secondary representations for certain modules over a commutative Noetherian ring}

The purpose of this section is to show that, if $E$ is an injective $A$-module and $M$ is a finitely generated $A$-module, then $\operatorname{Hom}_{A}(M, E)$ always has a secondary representation; we shall also give a precise description of the set $\operatorname{Att}_{A}\left(\operatorname{Hom}_{A}(M, E)\right)$ in terms of $\operatorname{Ass}_{A}(M)$ and $\operatorname{Occ}(E)$. The results of this section follow easily from work in [7].

Theorem 2.1. Let $E$ be an injective module over our commutative Noetherian ring $A$, and let $M$ be a finitely generated A-module. Then $\operatorname{Hom}_{A}(M, E)$ has a secondary representation, and, furthermore,

$$
\operatorname{Att}_{A}\left(\operatorname{Hom}_{A}(M, E)\right)=\left\{\mathfrak{p}^{\prime} \in \operatorname{Ass}_{A}(M): \mathfrak{p}^{\prime} \subseteq \mathfrak{p} \text { for some } \mathfrak{p} \in \operatorname{Occ}(E)\right\}
$$

Proof. In view of our conventions, the claims are clear when $M=0$, and so we shall assume that $M \neq 0$. Let $0=\bigcap_{i=1}^{n} Q_{i}$ be a minimal (that is normal) primary decomposition for the zero submodule of $M$, and, for each $i=1, \ldots, n$, let $\pi_{i}: M \rightarrow M / Q_{i}$ denote the natural epimorphism. Denote $\sqrt{ }\left(Q_{i}: M\right)$ by $\mathfrak{p}_{i}$ (for $\left.i=1, \ldots, n\right)$, so that $\operatorname{Ass}_{A}(M)=$ $\left\{\mathfrak{p}_{1}, \ldots, \mathfrak{p}_{n}\right\}$.

Let $T$ denote the exact, additive, contravariant, $A$-linear functor $\operatorname{Hom}_{A}(, E)$ from the category of all $A$-modules and $A$-homomorphisms to itself. For each $i=1, \ldots, n$, set

$$
S_{i}=T\left(\pi_{i}\right) T\left(M / Q_{i}\right)=\operatorname{Hom}_{A}\left(\pi_{i}, E\right) \operatorname{Hom}_{A}\left(M / Q_{i}, E\right)
$$

a submodule of $T(M)=\operatorname{Hom}_{A}(M, E)$. It follows from $[7,3.2]$ that $S_{i}$ is either zero or $\mathfrak{p}_{i}$-secondary (for $1 \leqq i \leqq n$ ), and that

$$
T(M)=\operatorname{Hom}_{A}(M, E)=S_{1}+S_{2}+\cdots+S_{n} .
$$

Thus $\operatorname{Hom}_{A}(M, E)$ has a secondary representation, and, if it is non-zero, we can arrive at a reduced secondary representation for it by first deleting any zero terms from the right 
hand side of the above equation, and then deleting redundant terms one at a time. Thus our proof will be complete once we have shown, for an integer $j$ between 1 and $n$, that (i) if, for every $\mathfrak{p} \in \operatorname{Occ}(E)$, we have $\mathfrak{p}_{j} \nsubseteq \mathfrak{p}$, then $\operatorname{Hom}_{A}\left(M / Q_{j}, E\right)=0$, and (ii) if $\mathfrak{p}_{j} \subseteq \mathfrak{p}^{\prime}$ for some $\mathfrak{p}^{\prime} \in \operatorname{Occ}(E)$, then

$$
\sum_{\substack{i=1 \\ i \neq j}}^{n} S_{i} \neq \operatorname{Hom}_{A}(M, E)
$$

We now prove these two claims in turn. We let $\left(\mathfrak{p}_{\alpha}\right)_{\alpha \in \Lambda}$ be a family of prime ideals of $A$ for which $E \cong \bigoplus_{\alpha \in \Lambda} E\left(A / \mathfrak{p}_{\alpha}\right)$.

(i) Since $M / Q_{j}$ is finitely generated,

$$
\operatorname{Hom}_{A}\left(M / Q_{j}, E\right) \cong \bigoplus_{\alpha \in \Lambda} \operatorname{Hom}_{A}\left(M / Q_{j}, E\left(A / \mathfrak{p}_{\alpha}\right)\right)
$$

We therefore show that $\operatorname{Hom}_{A}\left(M / Q_{j}, E\left(A / p_{\alpha}\right)\right)=0$ for each $\alpha \in \Lambda$. Now, for such an $\alpha$, we have $\mathfrak{p}_{j} \nsubseteq \mathfrak{p}_{\alpha}$ : choose $a \in \mathfrak{p}_{j} \backslash \mathfrak{p}_{\alpha}$. Then there exists $h \in \mathbb{N}$ such that $a^{h} \in\left(0: M / Q_{j}\right)$. But multiplication by $a^{h}$ provides an automorphism of $E\left(A / \mathfrak{p}_{\alpha}\right)$, by $[4$, Lemma 3.2(2)]. It therefore follows from the $A$-linearity of $\operatorname{Hom}_{A}($,$) that \operatorname{Hom}_{A}\left(M / Q_{j}, E\left(A / \mathfrak{p}_{\alpha}\right)\right)=0$. Thus point (i) has been established.

(ii) Set $K_{j}=\bigcap_{i \in J} Q_{i}$, where $J=\{1, \ldots, j-1, j+1, \ldots, n\}$. It follows from [7, 3.4] that $\operatorname{Hom}_{A}(M, E)=\sum_{i \in J} S_{i}$ if and only if $\operatorname{Hom}_{A}\left(K_{j}, E\right)=0$, and so it is enough for us to show that, if $\mathfrak{p}_{j} \subseteq \mathfrak{p}_{\alpha}$ for some $\alpha \in \Lambda$, then $\operatorname{Hom}_{A}\left(K_{j}, E\left(A / \mathfrak{p}_{\alpha}\right)\right) \neq 0$.

Now

$$
K_{j}=K_{j} / 0=K_{j} /\left(K_{j} \cap Q_{j}\right) \cong\left(K_{j}+Q_{j}\right) / Q_{j},
$$

so that $\operatorname{Ass}\left(K_{j}\right)=\left\{\mathfrak{p}_{j}\right\}$. Hence $K_{j}$ has a submodule isomorphic to $A / \mathfrak{p}_{j}$, and it is easy to use the injective property of $E\left(A / \mathfrak{p}_{\alpha}\right)$ in conjunction with the natural, non-zero, epimorphism $A / \mathfrak{p}_{j} \rightarrow A / \mathfrak{p}_{\alpha}$ to deduce that $\operatorname{Hom}_{A}\left(K_{j}, E\left(A / \mathfrak{p}_{\alpha}\right)\right) \neq 0$. This completes the proof of point (ii).

The result is now proved.

Corollary 2.2. Let $E$ be an injective module over our commutative Noetherian ring $A$, and let a be a proper ideal of $A$. Then $\left(0:_{E} \mathfrak{a}\right)$ has a secondary representation, and

$$
\operatorname{Att}_{A}\left(0::_{E} \mathfrak{a}\right)=\left\{\mathfrak{p}^{\prime} \in \text { ass } \mathfrak{a}: \mathfrak{p}^{\prime} \subseteq \mathfrak{p} \text { for some } \mathfrak{p} \in \operatorname{Occ}(E)\right\} .
$$

Proof. This is an immediate consequence of 2.1 because $\operatorname{Hom}_{A}(A / \mathfrak{a}, E) \cong(0: E a)$ and $\operatorname{Ass}_{A}(A / \mathfrak{a})=$ ass a. 


\section{The results}

To provide some motivation for our results, let us first consider an Artinian module $N$ over the commutative ring $R$, and let $I$ be an ideal of $R$. Every Artinian $R$-module possesses a secondary representation. In [8, Theorem 3.1], it was shown that both the sequences of sets

$$
\left(\operatorname{Att}_{R}\left(0:_{N} I^{n}\right)\right)_{n \in N} \text { and }\left(\operatorname{Att}_{R}\left(\left(0:_{N} I^{n+1}\right) /\left(0 i_{N} I^{n}\right)\right)\right)_{n \in N}
$$

are ultimately constant; let $\mathrm{At}^{*}(I, N)$ and $\mathrm{Bt}^{*}(I, N)$ (respectively) denote their ultimate constant values. Those results are, in a sense, dual to results which follow quickly from the work of Brodmann which was mentioned in the Introduction. It is easy to see (by, for example, $[3,(2.4)])$ that $\mathrm{Bt}^{*}(I, N) \subseteq \mathrm{At}^{*}(I, N)$. The second author proved in [9, Theorem (4.3)] that

$$
\operatorname{At}^{*}(I, N) \backslash \mathrm{Bt}^{*}(I, N) \subseteq \operatorname{Att}_{R}(N),
$$

and this result can be viewed as dual to a (natural generalization of a) result of McAdam and Eakin [5, Corollary 13]: see [9, Theorem (4.2)].

Now consider an injective module $E$ over our commutative Noetherian ring $A$, and let $a$ be an ideal of $A$. It follows from 2.2 that $\left(0:_{E} \mathfrak{a}^{n}\right)$ has a secondary representation (for every $n \in \mathbb{N}$ ), and so, by $[3,(2.4)]$, the $A$-module

$$
\left(0:_{E} \mathfrak{a}^{n+1}\right) /\left(0:_{E} \mathfrak{a}^{n}\right)
$$

has a secondary representation (for every $n \in \mathbb{N}$ ); we show below that the obvious analogues of the above-mentioned results for $N$ and $I$ hold for $E$ and $\mathfrak{a}$.

We shall use the following additional notation. For a finitely generated $A$-module $M$, we shall use $\operatorname{As}^{*}(a, M)$ and $\operatorname{Bs}^{*}(a, M)$ to denote the ultimate constant values of the sequences

$$
\left(\operatorname{Ass}_{A}\left(M / \mathfrak{a}^{n} M\right)\right)_{n \in N} \text { and }\left(\operatorname{Ass}_{A}\left(\mathfrak{a}^{n} M / \mathfrak{a}^{n+1} M\right)\right)_{n \in N}
$$

respectively: see [1].

Theorem 3.1. Let $E$ be an injective module over our commutative Noetherian ring $A$, and let $a$ be an ideal of $A$. Then the sequence of $\operatorname{sets}\left(\operatorname{Att}_{A}\left(0:_{E} \mathfrak{a}^{n}\right)\right)_{n \in N}$ is ultimately constant. We denote its ultimate constant value by At*(a,E). In fact,

$$
\operatorname{At}^{*}(\mathfrak{a}, E)=\left\{\mathfrak{p}^{\prime} \in \operatorname{As}^{*}(\mathfrak{a}, A): \mathfrak{p}^{\prime} \subseteq \mathfrak{p} \text { for some } \mathfrak{p} \in \operatorname{Occ}(E)\right\}
$$

Proof. By 2.2,

$$
\operatorname{Att}_{A}\left(0:_{E} \mathfrak{a}^{n}\right)=\left\{\mathfrak{p}^{\prime} \in \text { ass } \mathfrak{a}^{n}: \mathfrak{p}^{\prime} \subseteq \mathfrak{p} \text { for some } \mathfrak{p} \in \operatorname{Occ}(E)\right\}
$$


However, by Brodmann's work in [1], we have ass $a^{n}=\mathrm{As}^{*}(\mathfrak{a}, A)$ for all sufficiently large integers $n$, and the result follows immediately.

Theorem 3.2. Let $E$ be an injective module over our commutative Noetherian ring $A$, and let $a$ be an ideal of $A$. Then the sequence of sets

$$
\left(\operatorname{Att}_{A}\left(\left(0:_{E} \mathfrak{a}^{n+1}\right) /\left(0:_{E} \mathfrak{a}^{n}\right)\right)\right)_{n \in N}
$$

is ultimately constant. We denote its ultimate constant value by $\mathrm{Bt}^{*}(\mathfrak{a}, E)$. In fact,

$$
\mathrm{Bt}^{*}(\mathfrak{a}, E)=\left\{\mathfrak{p}^{\prime} \in \mathrm{Bs}^{*}(\mathfrak{a}, A): \mathfrak{p}^{\prime} \subseteq \mathfrak{p} \text { for some } \mathfrak{p} \in \operatorname{Occ}(E)\right\}
$$

Proof. We use a similar method to that employed above for 3.1. This time, we use, for $n \in \mathbb{N}$, the canonical short exact sequence

$$
0 \rightarrow \mathfrak{a}^{n} / \mathfrak{a}^{n+1} \rightarrow A / \mathfrak{a}^{n+1} \rightarrow A / \mathfrak{a}^{n} \rightarrow 0,
$$

the injective property of $E$, and the natural isomorphisms

$$
\operatorname{Hom}_{A}\left(A / \mathfrak{a}^{j}, E\right) \cong\left(0:_{E} a^{j}\right) \quad(j=n, n+1)
$$

to deduce that $\left(0:_{E} \mathfrak{a}^{n+1}\right) /\left(0:_{E} \mathfrak{a}^{n}\right) \cong \operatorname{Hom}_{A}\left(\mathfrak{a}^{n} / \mathfrak{a}^{n+1}, E\right)$. It therefore follows from [1] and 2.1 that, for all sufficiently large integers $n$,

$$
\operatorname{Att}_{A}\left(\left(0:_{E} \mathfrak{a}^{n+1}\right) /\left(0::_{E} \mathfrak{a}^{n}\right)\right)=\left\{\mathfrak{p}^{\prime} \in \mathrm{Bs}^{*}(\mathfrak{a}, A): \mathfrak{p}^{\prime} \subseteq \mathfrak{p} \text { for some } \mathfrak{p} \in \operatorname{Occ}(E)\right\}
$$

The claims of the theorem now follow immediately.

Corollary 3.3. Let $E$ be an injective module over our commutative Noetherian ring $A$, and let $a$ be an ideal of $A$. Then, with the notation of 3.1 and 3.2,

$$
\mathrm{Bt}^{*}(\mathfrak{a}, E) \subseteq \mathrm{At}^{*}(\mathfrak{a}, E) \quad \text { and } \quad \mathrm{At}^{*}(\mathfrak{a}, E) \backslash \mathrm{Bt}^{*}(\mathfrak{a}, E) \subseteq \mathrm{Att}_{A}(E)
$$

Proof. It follows from [3, (2.4)] that $\mathrm{Bt}^{*}(\mathfrak{a}, E) \subseteq \mathrm{At}^{*}(\mathfrak{a}, E)$. It is immediate from 3.1 and 3.2 that $\operatorname{At}^{*}(a, E) \backslash \mathrm{Bt}^{*}(a, E)$ is equal to the set

$$
\left\{\mathfrak{p}^{\prime} \in \mathrm{As}^{*}(\mathfrak{a}, A) \backslash \mathrm{Bs}^{*}(\mathfrak{a}, A): \mathfrak{p}^{\prime} \subseteq \mathfrak{p} \text { for some } \mathfrak{p} \in \operatorname{Occ}(E)\right\}
$$

But, by $\left[5\right.$, Corollary 13], $\operatorname{As}^{*}(\mathfrak{a}, A) \backslash \mathrm{Bs}^{*}(\mathfrak{a}, A) \subseteq \operatorname{Ass}_{A}(A)$. Hence

$$
\operatorname{At}^{*}(\mathfrak{a}, E) \backslash \mathrm{Bt}^{*}(\mathfrak{a}, E) \subseteq\left\{\mathfrak{p}^{\prime} \in \operatorname{Ass}_{A}(A): \mathfrak{p}^{\prime} \subseteq \mathfrak{p} \text { for some } \mathfrak{p} \in \operatorname{Occ}(E)\right\}
$$

However, the right hand set in the above display is, by [7, Theorem 2.6], just $\operatorname{Att}_{A}(E)$, and so the proof is complete. 


\section{REFERENCES}

1. M. Brodmann, Asymptotic stability of Ass $\left(M / I^{n} M\right)$, Proc. Amer. Math. Soc. 74 (1979), 16-18.

2. D. KIRBY, Coprimary decomposition of Artinian modules, J. London Math. Soc (2) 6 (1973), 571-576.

3. I. G. Macdonald, Secondary representation of modules over a commutative ring, Symposia Matematica 11 (Istituto Nazionale di alta Matematica, Roma, 1973), 23-43.

4. E. Matlis, Injective modules over Noetherian rings, Pacific J. Math. 8 (1958), 511-528.

5. S. McAdam and P. Eakin, The asymptotic Ass, J. Algebra 61 (1979), 71-81.

6. D. G. Northсотт, Generalized Koszul complexes and Artinian modules, Quart. J. Math. Oxford (2) 23 (1972), 289-297.

7. R. Y. SHARP, Secondary representations for injective modules over commutative Noetherian rings, Proc. Edinburgh Math. Soc. (2) 20 (1976), 143-151.

8. R. Y. Sharp, Asymptotic behaviour of certain sets of attached prime ideals, J. London Math. Soc. (2) 34 (1986), 212-218.

9. R. Y. Sharp, A method for the study of Artinian modules, with an application to asymptotic behavior, in Commutative algebra-proceedings of a microprogram held June 15-July 2, 1987 (Mathematical Sciences Research Institute Publications 15, Springer, New York, 1989), $443-465$.

Department of Pure Mathematics

UNIVERSITY OF SHEFFIELD

Hicks Building

SHEFFIELD S3 7RH 